\title{
Efficacy and Safety of Apatinib for Elderly Patients with Advanced or Metastatic Gastric Cancer After Failure of at Least First-Line Chemotherapy: A Multi-Center, Single-Arm, Phase II Study
}

Dengfeng Ren, (iD) ${ }^{1-3}$ Guoxiu Wang, ${ }^{4}$ Yu Zhang, ${ }^{5}$ Jie Kan, ${ }^{6}$ Qiuxia Dong, ${ }^{7}$ Junhui Zhao, ${ }^{3}$ Faxiang ji, ${ }^{3} \mathrm{Hao} \mathrm{Li}^{3}{ }^{3}$ Yushuang Luo, ${ }^{3}$ Mingzhe Lin, ${ }^{3}$ Guoyuan Li, ${ }^{3}$ Zhibo Liu, ${ }^{3}$ Xinfu Ma, ${ }^{8}$ Qijing Guo, ${ }^{3}$ Fuxing Zhao, ${ }^{1,2}$ Guoshuang Shen, ${ }^{2,8}$ jiuda Zhao ${ }^{1-3}$

'Research Center for High Altitude Medicine, Qinghai University, Xining, People's Republic of China; ${ }^{2}$ Breast Disease Diagnosis and Treatment Center, Affiliated Hospital of Qinghai University, Affiliated Cancer Hospital of Qinghai University, Xining, People's Republic of China; ${ }^{3}$ Department of Medical Oncology, Affiliated Hospital of Qinghai University, Affiliated Cancer Hospital of Qinghai University, Xining, People's Republic of China; ${ }^{4}$ Department of Medical Oncology, Yangquan No.I People's Hospital, Yangquan, People's Republic of China; ${ }^{5}$ Department of Medical Oncology, Qinghai Red Cross Hospital, Xining, People's Republic of China; ${ }^{6}$ Department of Medical Oncology, People's Hospital of Qinghai Province, Xining, People's Republic of China; ${ }^{7}$ Department of Medical Oncology, The Fifth People's Hospital of Qinghai Province, Xining, People's Republic of China; ${ }^{8}$ Department of Surgical Oncology, Affiliated Hospital of Qinghai University, Affiliated Cancer Hospital of Qinghai University, Xining, People's Republic of China

Correspondence: Jiuda Zhao

Research Center for High Altitude Medicine, Qinghai University, Xining, 810000, People's

Republic of China

Tel +86 1332766 1976

Email jiudazhao@I26.com

Guoshuang Shen

Breast Disease Diagnosis and Treatment

Center, Affiliated Hospital of Qinghai

University, Affiliated Cancer Hospital of

Qinghai University, Xining, 810000, People's

Republic of China

Tel +86 13109708777

Email guoshuangshen@I26.com
Background: Apatinib improves progression-free survival and overall survival with an acceptable safety profile in Chinese patients with chemotherapy-refractory advanced or metastatic gastric cancer. However, the efficacy and safety of apatinib are unclear for elderly patients. This study was undertaken to prospectively investigate the efficacy and safety of apatinib for elderly patients with unresectable advanced or metastatic gastric cancer, who experienced progression to at least one lines of chemotherapy.

Methods: This open-label, single-arm, phase II study enrolled patients aged $\geq 60$ years with advanced gastric cancer, who experienced progression to one or more lines of chemotherapy at five centers in China. Patients received apatinib in an oral dose of $500 \mathrm{mg}$ or $250 \mathrm{mg}$ daily according to the research physicians' decision. The primary end point was progression-free survival, and the secondary end points were objective response rate, disease control rate, overall survival, and safety.

Results: Forty-eight patients were enrolled between June 2017 and September 2019. The median age was 65.5 years (range 60-80 years). Twenty-seven patients (56.3\%) started treatment with an initial dose of $500 \mathrm{mg}$ and 21 patients $(43.7 \%)$ with $250 \mathrm{mg}$. The median progression-free survival and overall survival were 3.00 months (95\% confidence interval, 2.17-3.84) and 8.10 months (95\% confidence interval, 4.35-11.85), respectively. The objective response rate and disease control rate assessed by the investigators were $16.7 \%$ and $72.9 \%$, respectively. The common side effects were fatigue $(58.3 \%)$, hypertension $(47.9 \%)$, abdominal pain (33.3\%), proteinuria (29.2\%), leukopenia $(22.9 \%)$, and neutropenia $(20.8 \%)$. Hypertension (22.9\%) was the major grade $3 / 4$ toxicity.

Conclusion: These data suggest that apatinib is effective and relatively tolerable for elderly patients with unresectable advanced or metastatic gastric cancer who have received at least first-line chemotherapy.

Keywords: apatinib, efficacy, safety, elderly patients, advanced gastric cancer

\section{Introduction}

According to the newest global cancer statistics, gastric cancer (GC) is the fifth most commonly diagnosed cancer and the fourth leading cause of cancer death worldwide. ${ }^{1}$ Despite the slight decline of incidence, Eastern Asia still has the highest GC incidence rates, and because of the absence of early specific symptoms, $80 \%$ of patients in China are diagnosed at advanced stages, with a low 5-year survival rate. $^{2}$ In the past thirty years, fluorouracil- and platinum-based 
chemotherapies were widely used in advanced or metastatic GC, which improved the survival of patients by about half a year. However, the improvement of survival is limited by the finite chemotherapeutic drugs and toxicity caused by chemotherapy.

Nowadays, targeted therapeutic agents, including monoclonal antibodies and tyrosine kinase inhibitors (TKIs), have been shown to improve progression-free survival (PFS) or overall survival (OS) of advanced, recurrent, or metastatic gastric or gastroesophageal junction (GEJ) cancers by blocking the molecular signaling pathway for tumor growth, such as human epidermal growth factor receptor 2 (HER2), programmed cell death protein 1 (PD-1), and vascular endothelial growth factor receptor (VEGFR). ${ }^{3-6}$ Up to now, three FDA-approved targeted drugs, trastuzumab, pembrolizumab, and ramucirumab, have been used in advanced GC..$^{7-11}$ Trastuzumab, a HER2 monoclonal antibody, combined with chemotherapy as a first-line regimen significantly improved PFS and OS in HER2-positive advanced gastric or GEJ cancer, establishing the first targeted therapy in GC patients. ${ }^{3}$ Pembrolizumab, a PD-1 antibody, was demonstrated effective with an ORR from $15.5 \%$ to $22 \%$ in PD-L1-positive gastric or GEJ adenocarcinoma patients who had progressed following two or more prior lines of therapy and acquired the similar OS, but a better safety profile, compared with that of standard-dose paclitaxel in second-line therapy. ${ }^{4,12,13}$ Moreover, antiangiogenic agents have shown promising efficacy to date. Although the antiVEGF monoclonal antibody bevacizumab failed to improve OS in advanced $\mathrm{GC},{ }^{14}$ ramucirumab, a fully human anti-VEGFR-2 monoclonal antibody, and apatinib, a novel VEGFR2 TKI, were proved to prolong both PFS and OS in patients with advanced GC. . $^{5,6,15,16}$

Apatinib is an orally administered small-molecule VEGFR2 TKI that has been proved effective and safe in patients with chemotherapy-refractory advanced or metastatic GC. Previous phase II and III clinical trials indicated that compared to placebo, apatinib significantly improved PFS (3.67 vs 1.4 months, $p<0.001 ; 2.6$ vs 1.8 months, $p<0.001)$ and OS (4.83 vs 2.5 months, $p<0.001 ; 6.5$ vs 4.7 months, $p=0.0149$ ) in patients with advanced or metastatic adenocarcinoma of the stomach or GEJ. ${ }^{6,16}$ Apatinib was subsequently approved in China for advanced or metastatic GC refractory to two or more lines of prior chemotherapy in 2014 and was considered as a standard third-line therapy in China. ${ }^{17,18}$
Nowadays, with the aging of the population, elderly people are more likely to be diagnosed with GC owing to the increased life expectancy. ${ }^{1}$ In China, approximately $68.6 \%(465,900 / 679,100)$ of GCs occurred in patients over the age of 60 , and they account for about $76.1 \%$ $(379,000 / 498,000)$ of GC deaths. ${ }^{19}$ However, because of the comorbidities or age-related changes in pharmacokinetics and pharmacodynamics that may result in higher toxicity and increased risk of death, the inclusion of elderly patients in RCT is often hampered. ${ }^{20}$ In the phase II trial of apatinib, the median age of enrolled patients was 55 years and 53 years in two apatinib groups, which means only a few participants were elderly patients. ${ }^{16}$ Moreover, there were just 21 elderly GC patients (>65 years) included in the apatinib group $(n=176)$ with a median age of 58 years in the phase III clinical trial, and a subgroup analysis revealed no clinical benefit of apatinib in those elderly patients. ${ }^{6}$ Because of the limited number of elderly patients included, whether apatinib was effective and safe for elderly patients was still not clear. Hence, we conducted this multicenter, single-arm, phase II study to further investigate the efficacy and safety of apatinib in elderly patients with unresectable advanced or metastatic GC, progressed with or could not tolerate one or more lines of chemotherapy.

\section{Materials and Methods Study Design and Patients}

This multicenter, single-arm, phase II clinical trial was conducted at five institutions in China. The aim of the study was to evaluate the efficacy and safety of apatinib in elderly patients with unresectable advanced or metastatic GC, who progressed with or could not tolerate one or more lines of chemotherapy. The inclusion criteria comprised patients with histologically confirmed advanced or metastatic adenocarcinoma of the stomach or GEJ; age of 60 years or older upon enrollment; an Eastern Cooperative Oncology Group (ECOG) performance status (PS) of 0-2; progression with or intolerance to one or more lines of chemotherapy; at least one measurable lesion as defined by the Response Evaluation Criteria in Solid Tumors (RECIST) version 1.1; acceptable hematologic, cardiac, hepatic, pulmonary, and renal function; and estimated life expectancy $\geq 3$ months. Patients with any of the following conditions were excluded: difficulty in taking apatinib orally; uncontrolled central nervous system metastases; massive hydrothorax or ascites; proteinuria 2 
+ or 24-hour urinary protein $\geq 1 \mathrm{~g}$; recent traumatism or pathological fracture; uncontrolled blood pressure on medication ( $\geq 140 / 90 \mathrm{mmHg}$ ); and chemotherapy in the past 28 days before enrollment. Patients with bleeding tendency; receiving thrombolytics or anticoagulants; receiving intravenous antibiotic treatment; who had received ramucirumab, bevacizumab, or other VEGF TKIs before; or with other primary malignancy (except basal cell skin cancer or cervical carcinoma in situ) were also excluded.

\section{Treatment Plan}

Patients received apatinib (Jiangsu Hengrui Medicine Co., Lianyungang, China) $250 \mathrm{mg}$ or $500 \mathrm{mg}$ orally at a fixed time, once a day, which was decided by the investigators according to the patients' physical condition. Four weeks were defined as one treatment cycle. Dose reduction from $500 \mathrm{mg}$ to $250 \mathrm{mg}$, treatment interruption ( $\leq 14$ days), and supportive therapy were allowed when severe adverse events (AEs) happened, and dose reescalation was also permitted when AEs were relieved. The treatment continued until the patients experienced disease progression or intolerable toxicity or withdrew informed consent.

\section{Efficacy and Safety Assessment}

The primary end point of our study was PFS, which was defined as the time from enrollment to disease progression or death. The secondary end points included objective response rate (ORR), disease control rate (DCR), OS, and safety. Objective response was defined as complete response (CR) or partial response (PR), and disease control included $\mathrm{CR}, \mathrm{PR}$, and stable disease (SD) according to RECIST guidelines (version 1.1). OS was calculated from enrollment to death from any cause. Physical examination, complete blood count (CBC), blood biochemistry, and contrast-enhanced computed tomography (CT) scan of the chest, abdomen, and pelvis were performed at baseline. Subsequently, physical examination and CBC were conducted every 2 weeks; blood biochemistry including hepatic and renal function tests was performed every 4 weeks. Tumor response to treatment was evaluated by contrastenhanced CT scan every two treatment cycles ( 8 weeks) by the local investigators of each center.

All AEs were evaluated using the National Cancer Institute Common Terminology Criteria for Adverse Events version 4.0. For the first treatment cycle, toxicity was assessed every week and then biweekly during the following cycles.

\section{Ethical Permission}

This study was approved by the institutional research ethics committee of affiliated hospital of Qinghai University and conducted in accordance with the standards of the International Conference on Harmonization Good Clinical Practice Guidelines and the principles of the Helsinki Declaration of 1964 and later versions. Written informed consent was obtained from all patients prior to study enrollment. In addition, this study was registered at clinicaltrials.gov (identifier: NCT03104283).

\section{Statistical Analysis}

To calculate the sample size, we referred to the results of phase II and phase III clinical trials of apatinib. ${ }^{6,16}$ Accordingly, the median PFS (mPFS) was 2.6-3.67 months in the apatinib group. The expected mPFS in our study was 3.0 months. As a result, at a one-sided significance level of $5 \%$ and a power of $80 \%$ and assuming a dropout rate of $10 \%$ with a recruiting time of 2 years and 1-year follow-up time, at least 47 patients were required for our study.

The mPFS and median OS (mOS) were estimated using the Kaplan-Meier method, and Greenwood's formula was used to calculate the $95 \%$ confidence interval (CI) of the survival rate. All statistical analyses were performed using SPSS software version 21.0 (SPSS Inc., Chicago, IL, United States). All $p$ values were two sided, and $p<0.05$ was considered as statistically significant.

\section{Results}

\section{Patient Characteristics}

Forty-eight patients with unresectable advanced or metastatic gastric or GEJ adenocarcinoma were enrolled from five institutions between June 2017 and September 2019 in China. Patient characteristics are listed in Table 1. The median age of all included patients was 65.5 years (range 60-80 years), and most patients were males $(83.3 \%)$ and with metastatic disease $(89.6 \%)$. About half of the patients had an ECOG performance status score of 2 and more than two sites of metastasis, of which liver (52.1\%) and lymph node $(47.9 \%)$ metastases were more common.

\section{Treatment Delivery}

All enrolled patients received at least one cycle of apatinib. The median number of treatment cycles was 3.62 (range 1.04-25.46) and the number of average 
Table I Patient Demographics and Clinical Characteristics

\begin{tabular}{|c|c|c|}
\hline Characteristic & Number & Percentage (\%) \\
\hline \multicolumn{3}{|l|}{ Age (years) } \\
\hline $60-65$ & 16 & 33.3 \\
\hline$\geq 65$ & 32 & 66.7 \\
\hline \multicolumn{3}{|l|}{ Gender } \\
\hline Male & 40 & 83.3 \\
\hline Female & 8 & 16.7 \\
\hline \multicolumn{3}{|l|}{ ECOG performance status } \\
\hline 0 & $\mathrm{I}$ & 2.1 \\
\hline 1 & 23 & 47.9 \\
\hline 2 & 24 & 50.0 \\
\hline \multicolumn{3}{|l|}{ Primary tumor site } \\
\hline Gastric & 29 & 60.4 \\
\hline Gastroesophageal junction & 19 & 39.6 \\
\hline \multicolumn{3}{|l|}{ Prior surgery of primary lesion } \\
\hline Yes & 21 & 43.8 \\
\hline No & 27 & 56.2 \\
\hline \multicolumn{3}{|l|}{ Clinical staging } \\
\hline III & 5 & 10.4 \\
\hline IV & 43 & 89.6 \\
\hline \multicolumn{3}{|l|}{ No. of metastatic sites } \\
\hline$<2$ & 24 & 50.0 \\
\hline$\geq 2$ & 24 & 50.0 \\
\hline \multicolumn{3}{|l|}{ Metastatic sites } \\
\hline Liver & 25 & 52.1 \\
\hline Lung & 7 & 14.6 \\
\hline Lymph node & 23 & 47.9 \\
\hline Peritoneum & 6 & 12.5 \\
\hline Bone & 2 & 4.2 \\
\hline Others (spleen, ovary et al) & 13 & 27.1 \\
\hline \multicolumn{3}{|l|}{ No. of previous chemotherapy lines } \\
\hline I & 35 & 72.9 \\
\hline$\geq 2$ & 13 & 27.1 \\
\hline \multicolumn{3}{|l|}{ Prior radiotherapy } \\
\hline Yes & 3 & 6.2 \\
\hline No & 45 & 93.8 \\
\hline \multicolumn{3}{|l|}{ History of hypertension } \\
\hline Yes & 12 & 25.0 \\
\hline No & 36 & 75.0 \\
\hline \multicolumn{3}{|l|}{ Initial dose (mg) } \\
\hline 250 & 21 & 43.7 \\
\hline 500 & 27 & 56.3 \\
\hline
\end{tabular}

Abbreviation: ECOG, Eastern Cooperative Oncology Group.

treatment cycles was 5.98 (range 1.04-25.46). Twentyseven patients $(56.3 \%)$ started the treatment with an initial dose of $500 \mathrm{mg}$, and nine of them experienced dose reduction from $500 \mathrm{mg}$ to $250 \mathrm{mg}$ because of drugrelated grade 3 AEs. Others started with a dose of $250 \mathrm{mg}$, according to the researchers, until disease progression. Thirteen patients $(27.1 \%)$ received local therapy or late-line chemotherapy after the progression of apatinib treatment.

\section{Efficacy}

The median follow-up time was 7.62 months. The mPFS was 3.00 months (95\% CI, 2.17-3.84) (Figure 1), and mOS was 8.10 months (95\% CI, 4.35-11.85) (Figure 2). The one-year survival rate was $22.9 \%$. ORR assessed by the investigators was $16.7 \%$, and DCR was $72.9 \%$ (Table 2).

Exploratory subgroup analysis showed that for patients receiving apatinib at $250 \mathrm{mg}$ and $500 \mathrm{mg}$, the mPFS was 3.47 months and 2.80 months, respectively $(p=0.385)$, and the mOS was 8.13 months and 5.40 months, respectively $(p=0.244)$. There was no significant difference in mPFS between patients with different ECOG PS scores ( $0-1$ vs 2$)$, different age groups $(\geq 65$ vs 60-65), whether with AEs in the first 4 weeks, and whether with liver metastasis. Patients with ECOG PS score of $0-1$ live much longer than those with ECOG PS scores of 2 with a mOS of 9.60 months and 3.83 months $(p=0.007)$, respectively. However, there was no significant difference in mOS between patients with different ages ( $\geq 65$ vs $60-65$ ), whether with AEs in the first 4 weeks and whether with liver metastasis (Figure 3A-J).

\section{Safety}

Treatment-related AEs of different grades are shown in Table 3. The most common AEs of grade 3 or 4 were hypertension (22.9\%) followed by neutropenia (4.2\%) and mucosal inflammation (4.2\%). Fatigue was the most common AEs of any grade, with an incidence of $58.3 \%$. Other common AEs of any grade included hypertension (47.9\%), abdominal pain (33.3\%), proteinuria (29.2\%), leukopenia (22.9\%), and neutropenia $(20.8 \%)$. The incidence rates of any grade hypertension in patients with or without a history of hypertension were $83.3 \%(10 / 12)$ and $36.1 \%(13 / 36)$, respectively. And the incidence rates of grade 3 to 4 hypertension were $50.0 \%(6 / 12)$ and $13.9 \%(5 / 36)$ in these two groups of patients.

Moreover, three patients experienced various degrees of gastrointestinal bleeding while on medicine. Nine 


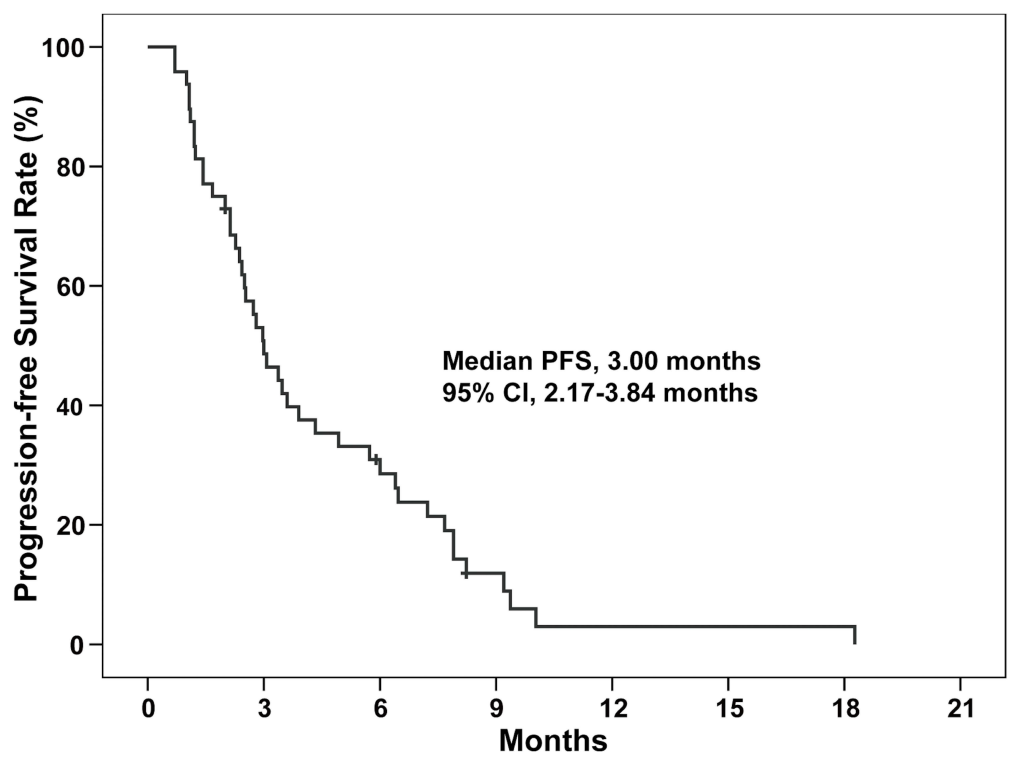

Figure I Kaplan-Meier plot of progression-free survival.

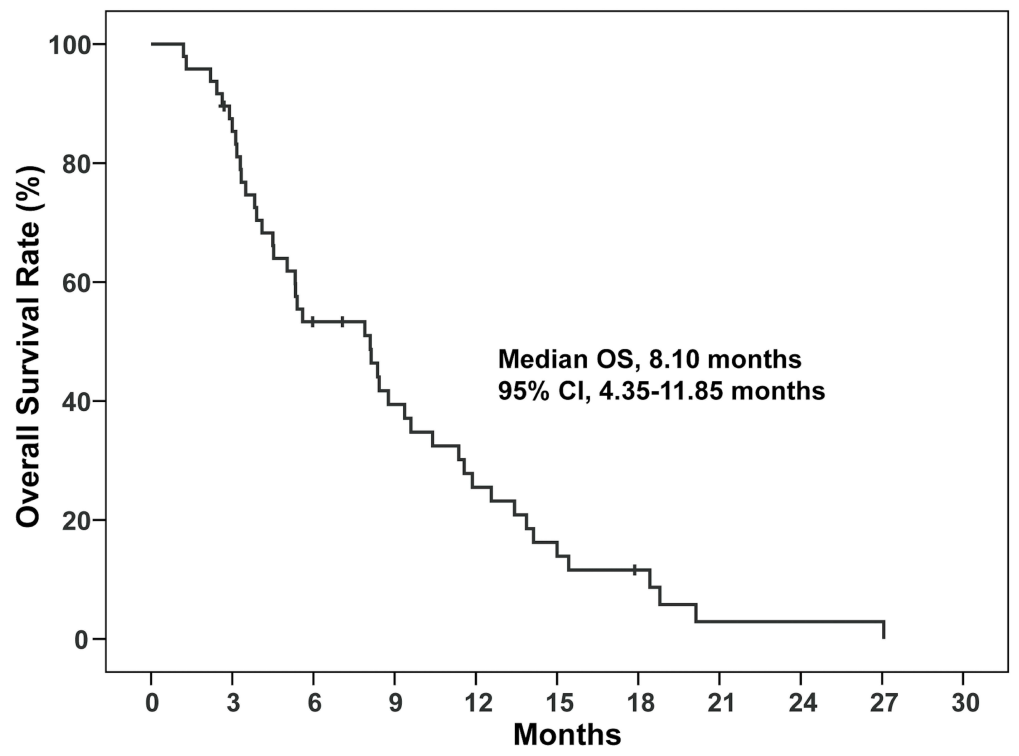

Figure 2 Kaplan-Meier plot of overall survival.

patients $(18.8 \%)$ experienced dose reduction because of hypertension $(\mathrm{n}=3)$, upper gastrointestinal hemorrhage $(\mathrm{n}=2)$, fatigue $(\mathrm{n}=2)$, mucosal inflammation $(\mathrm{n}=1)$, and hand-foot syndrome $(n=1)$. There was no drug-related mortality.

\section{Discussion}

To the best of our knowledge, this is the first prospective clinical trial that aims to investigate the efficacy and safety of apatinib in elderly GC patients.
The 3.00-month mPFS showed that this study met the primary end point previously determined. In addition, the ORR and DCR were relatively high $(16.7 \%$ and $72.9 \%$, respectively), and the median OS was as long as 8.10 months. Moreover, apatinib was welltolerated with manageable toxicities in elderly patients with GC.

As it is known, the incidence of gastric or GEJ adenocarcinoma increases with age. According to a Stomach cancer Pooling (StoP) project, the median 
Table 2 Tumor Responses (N=48) Based on RECIST (Version I.I)

\begin{tabular}{|l|c|}
\hline Responses & n (\%) \\
\hline Complete response & $0(0.0)$ \\
Partial response & $8(16.7)$ \\
Stable disease & $27(56.2)$ \\
Progressive disease & $13(27.1)$ \\
Overall response rate & 16.7 \\
Disease control rate & 72.9 \\
\hline
\end{tabular}

Abbreviations: RECIST, Response Evaluation Criteria in Solid Tumor; Cl, confidence interval.

age for patients with GC is 61 years, which means half of the GC patients are older than 61 years. ${ }^{21}$ However, owing to the strict selection and underrepresentation of elderly patients in clinical trials, there are limited data concerning GC treatment in this specific age group. ${ }^{22,23}$ Most of the data about elderly GC patients are from subgroup analyses of randomized clinical trials or small phase II studies. Moreover, elderly patients included in clinical trials tend to be in good physical condition, and those with comorbidities are usually excluded. Furthermore, elderly patients are usually associated with progressive reduction in functional reserve, more chronic diseases, and changes of pharmacokinetics and pharmacodynamics, which may lead to higher toxicity and more risk of treatment-related death. $^{24}$

Although there are limited data available regarding the efficacy and safety of angiogenesis-targeted agents in elderly patients with advanced GC, ramucirumab has been proved effective and safe for elderly patients with advanced gastric or GEJ adenocarcinoma in the subgroup analyses of two randomized phase III trials. ${ }^{5,15}$ In the randomized phase III RAINBOW trial, the addition of ramucirumab to paclitaxel resulted in a significant improvement in PFS (HR 0.673, 95\% CI $0.506-0.894)$ in patients equal to or greater than 65 years old with a higher rate of grade 3 or 4 neutropenia (49\% vs $24 \%) .{ }^{15}$ The REGARD trial revealed that compared with placebo, the monotherapy of ramucirumab for previously treated advanced gastric or GEJ adenocarcinoma also offered a benefit in PFS (HR $0.49,95 \%$ CI $0.319-0.752$ ) in patients $\geq 65$ years old. ${ }^{5}$ In addition, Subpopulation Treatment Effect Pattern Plot (STEPP) analysis of RAINBOW and REGARD studies indicated that ramucirumab conferred improvements in efficacy across age groups $(\leq 45$ years,
$<65$ years, $\geq 65$ years, and $\geq 70$ years), with similar toxicity. ${ }^{25}$

Moreover, phase II and III clinical trials of apatinib have indicated its efficacy and safety in patients with chemotherapy-refractory advanced or metastatic adenocarcinoma of the stomach or GEJ. ${ }^{6,16} \mathrm{GC}$ patients aged between 18 years and 70 years were included in these two clinical trials. The median age of enrolled patients in the phase II study was 53 years and 55 years in two apatinib groups, which means that just a small amount of elderly patients were included, and in the phase III trial, only $12 \%(21 / 176)$ of patients who received the apatinib treatment were older than 65 years, and the subgroup analysis showed no PFS benefit compared with that in placebo, which might be attributed to the limited number of elderly patients. ${ }^{6,16}$ Hence, whether elderly patients would benefit from apatinib was not clear until now.

In our study, all enrolled patients were equal to or older than 60 years and the mPFS met the primary end point previously determined. The PFS benefits (mPFS: 3.00 months [95\% CI, 2.17-3.84]) in our study were better than those in the REGARD trial with ramucirumab monotherapy for previously treated advanced gastric or GEJ adenocarcinoma (mPFS: 2.1 months [interquartile range 1.3-4.2]) and the phase III study of apatinib monotherapy for those who experienced at least two lines of chemotherapy failure (mPFS, 2.6 months [95\% CI 2.0-2.9]). ${ }^{5,6}$ The mOS of all patients was also higher than that of the phase III trial (8.10 months; $95 \%$ CI, 4.35-11.85] vs 6.5 months; 95\% CI, 4.8-7.6). Moreover, patients in our study also had a higher ORR and DCR than those in the phase III trial $(16.7 \%$ vs $2.84 \% ; 72.9 \%$ vs $42.05 \%$ ). A possible reason might be that most patients $(72.9 \%)$ in our study only received first-line chemotherapy. In addition, the global phase III study of apatinib, ANGEL trial, showed that mPFS, ORR, and DCR were significantly improved with apatinib (2.83 months vs 1.77 months, $\mathrm{HR}=0.57,95 \% \mathrm{CI}$ $0.46-0.79, p<0.0001 ; 6.87 \%$ vs $0 \%, p=0.0020 ; 42.37 \%$ vs $13.08 \%, p<0.0001)$ in patients which were similar with the phase III trial in Chinese patients. ${ }^{26}$ In this global study, although OS was not significantly improved in the overall population with advanced/metastatic GC who failed $\geq 2$ prior chemotherapy regimens, most other efficacy endpoints including OS in patients received $\geq 4$ th line treatment, PFS, ORR, and DCR 

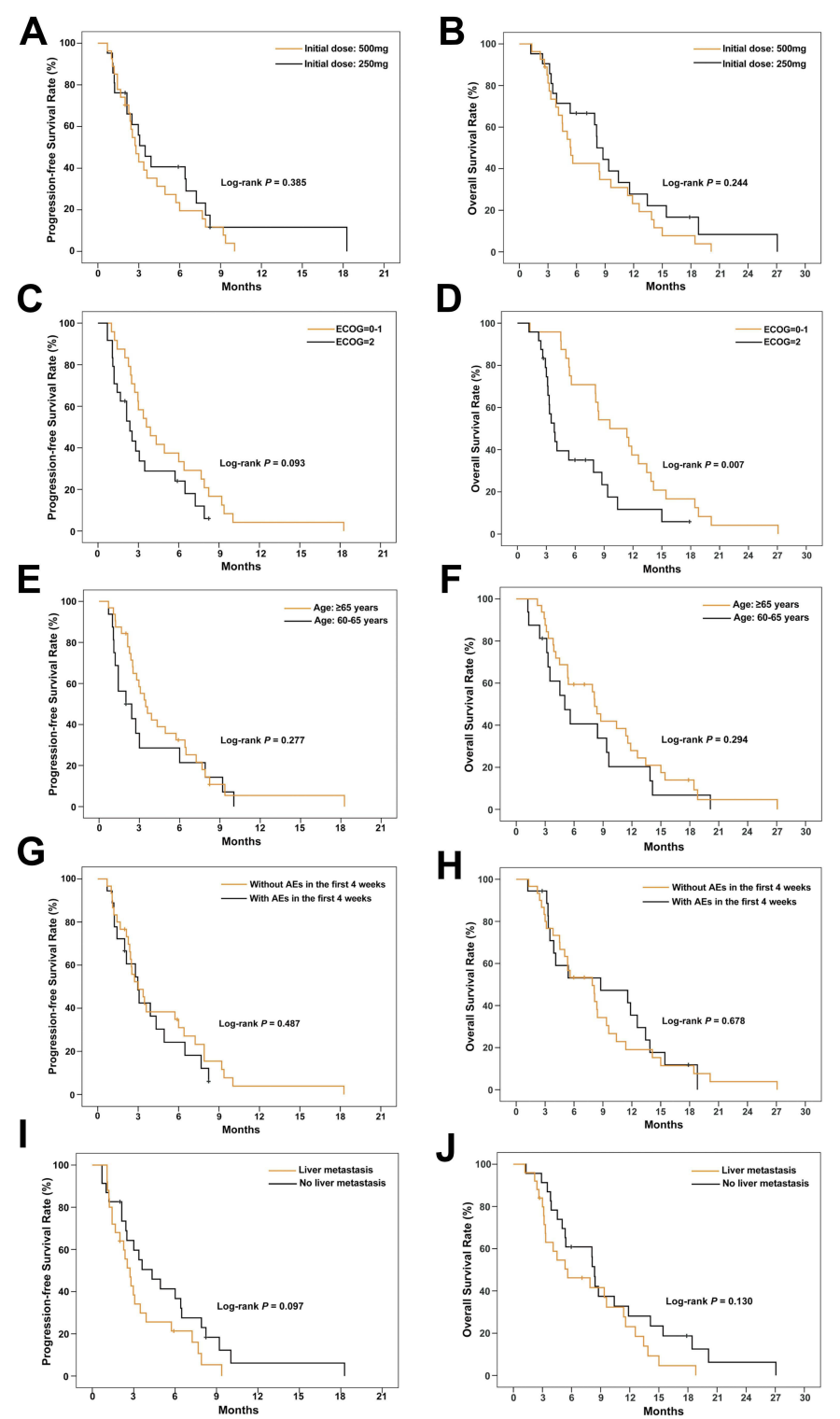

Figure 3 Kaplan-Meier plots of progression-free survival and overall survival of different subgroups. Plots (A and $\mathbf{B})$ show progression-free survival and overall survival according to different initial dose (500 mg vs $250 \mathrm{mg}$ ) of apatinib. Plots (C and D) show progression-free survival and overall survival according to different ECOG scores (0I vs 2). Plots (E and $\mathbf{F}$ ) show progression-free survival and overall survival according to different ages ( $\geq 65$ years vs $60-65$ years). Plots ( $\mathbf{G}$ and $\mathbf{H})$ show progression-free survival and overall survival according to whether any grade of AEs (including hypertension, proteinuria and hand-foot syndrome) happened during the first 4 weeks of medication. Plots ( $\mathbf{I}$ and $\mathbf{J})$ show progression-free survival and overall survival according to whether there is liver metastasis.

suggest that apatinib is effective and generally well tolerated. $^{26}$

Regarding the oral dose of apatinib, although the recommended dosage was $850 \mathrm{mg}$ once daily according to two key studies of apatinib, the dose of $250-850 \mathrm{mg}$ was widely used by attending oncologists in China. Recently, a real-world study of apatinib in advanced GC patients in China (AHEAD-G202) with 337 patients included showed that majority $(95.6 \%)$ of patients received the dose of $250-500 \mathrm{mg}$ and only $15(4.4 \%)$ patients took $675-850 \mathrm{mg}$ as the starting dose. ${ }^{27}$ Moreover, the sub-analysis of the AHEADG202 study revealed no significant difference in PFS and OS among the low- $(250 \mathrm{mg})$, mid- (425$500 \mathrm{mg})$, and high-dose $(625-850 \mathrm{mg})$ groups. $^{28} \mathrm{In}$ our study, the initial dosage of apatinib was $250 \mathrm{mg}$ or $500 \mathrm{mg}$ once daily at the discretion of the investigators. Our study also showed that there was no difference in mPFS (3.47 months vs 2.80 months, $p=$ $0.385)$ and mOS (8.13 months vs 5.40 months, $p=$ 0.244 ) between patients receiving dosage of $250 \mathrm{mg}$ and $500 \mathrm{mg}$. 
Table 3 Adverse Events ( $\mathrm{N}=48$ ) Based on CTCAE (Version 4.0)

\begin{tabular}{|l|c|c|c|c|c|c|}
\hline \multirow{2}{*}{ Adverse Events } & \multicolumn{4}{|c|}{ Grade } & \multicolumn{1}{c|}{ Total } & Grade 3/4 \\
\cline { 2 - 7 } & I & $\mathbf{2}$ & $\mathbf{3}$ & $\mathbf{4}$ & $\mathbf{n}(\%)$ & $\mathbf{n}(\%)$ \\
\hline Hypertension & 4 & 8 & $\mathrm{II}$ & 0 & $23(47.9)$ & $\mathrm{II}(22.9)$ \\
Proteinuria & 12 & 2 & 0 & 0 & $14(29.2)$ & $0(0.0)$ \\
Hand-foot syndrome & 5 & 3 & 0 & 0 & $8(16.7)$ & $0(0.0)$ \\
Leukopenia & 5 & 5 & $\mathrm{I}$ & 0 & $11(22.9)$ & $\mathrm{I}(2.1)$ \\
Neutropenia & 5 & 3 & 2 & 0 & $10(20.8)$ & $2(4.2)$ \\
Anemia & 6 & 3 & 0 & 0 & $9(18.7)$ & $0(0.0)$ \\
Thombocytopenia & 4 & 2 & $\mathrm{I}$ & 0 & $7(14.6)$ & $\mathrm{I}(2.1)$ \\
Elevated transaminase & 8 & 0 & $\mathrm{I}$ & 0 & $9(18.7)$ & $\mathrm{I}(2.1)$ \\
Bleeding & $\mathrm{I}$ & $\mathrm{I}$ & $\mathrm{I}$ & 0 & $3(6.2)$ & $\mathrm{I}(2.1)$ \\
Nausea & 5 & 2 & 0 & 0 & $7(14.6)$ & $0(0.0)$ \\
Vomiting & 8 & 0 & 0 & 0 & $8(16.7)$ & $0(0.0)$ \\
Diarrhea & 5 & 3 & 0 & 0 & $8(16.7)$ & $0(0.0)$ \\
Fatigue & 14 & 14 & 0 & 0 & $28(58.3)$ & $0(0.0)$ \\
Abdominal pain & $\mathrm{II}$ & 4 & $\mathrm{I}$ & 0 & $16(33.3)$ & $\mathrm{I}(2.1)$ \\
Mucosal inflammation & 5 & $\mathrm{I}$ & 2 & 0 & $8(16.7)$ & $2(4.2)$ \\
\hline
\end{tabular}

Abbreviation: CTCAE, Common Terminology Criteria for Adverse Events.

Despite the inspiring improvement of PFS and OS, apatinib has also caused various AEs to GC patients, which might reduce the quality of their life. For antiangiogenic therapy, hypertension, proteinuria, handfoot syndrome, and fatigue are the most common nonhematologic AEs. In our study, the most common AE of any grade was fatigue (58.3\%), and the second was hypertension $(47.9 \%)$, which were much higher compared with that of previous clinical trials ${ }^{6,16}$ in spite of the low dosage of apatinib. This might be partly because of the elderly patients among whom $25 \%$ had essential hypertension. The result of our study showed that compared to patients without a history of hypertension, patients with essential hypertension developed more hypertension of any grade (83.3\% vs $36.1 \%)$ and grade 3 to $4(50.0 \%$ vs $13.9 \%)$. In addition, less proteinuria (29.2\%), hand-foot syndrome (16.7\%), and hematologic toxicities were observed, which could be attributed to the low dose. Notably, eight patients (16.7\%) experienced mucosal inflammation of different grades.

Our study has several limitations. First, this study was a phase II, single-arm study with a limited sample size. Second, the study was only conducted in Chinese patients, so the generalization of apatinib to GC patients of other populations is limited. Third, we selected 60 years of age as the threshold of elderly patients which might be different from that of some other studies.
In conclusion, our study indicates that apatinib is effective in elderly patients with unresectable advanced or metastatic gastric or GEJ adenocarcinoma, who have already experienced one or more lines of chemotherapy. The most common side effects of apatinib for elderly patients were fatigue and hypertension. Given that the sample size of this study was small, further investigation with much more participants is warranted for verification.

\section{Data Sharing Statement}

The authors intend to share individual de-identified participant data. All data and study documents related to this study are always available from the corresponding authors (jiudazhao@126.com and guoshuangshen@126.com) for researchers engaged in related research on reasonable request after the publication of the primary manuscript.

\section{Acknowledgments}

We would like to thank Dr. Fangchao Zheng (Department of Medical Oncology, National Cancer Center/Cancer Hospital, Chinese Academy of Medical Sciences and Peking Union Medical College, Beijing, China) for valuable advice to this manuscript and Professor Li Dong (Department of Epidemiology and Medical Statistics, Shanxi University, Taiyuan, China) for help on statistics.

\section{Author Contributions}

All authors made a significant contribution to the work reported, whether that is in the conception, study design, execution, acquisition of data, analysis and interpretation, or in all these areas; took part in drafting, revising or critically reviewing the article; gave final approval of the version to be published; have agreed on the journal to which the article has been submitted; and agree to be accountable for all aspects of the work.

\section{Funding}

This work was supported by Beijing Xisike Clinical Oncology Research Foundation (Y-HR2016-024) and Project of Basic Research for application of Science and Technology Department of Qinghai Province (2019-ZJ -7003).

\section{Disclosure}

The authors declare no potential conflicts of interest in this work. 


\section{References}

1. Hyuna S, Jacques F, Siegel Rebecca L, et al. Global cancer statistics 2020: GLOBOCAN estimates of incidence and mortality worldwide for 36 cancers in 185 countries. CA Cancer J Clin. 2021;71 (3):209-249. doi:10.3322/caac.21660

2. Zong L, Abe M, Seto Y, Ji J. The challenge of screening for early gastric cancer in China. Lancet. 2016;388(10060):2606. doi:10.1016/ S0140-6736(16)32226-7

3. Bang YJ, Van Cutsem E, Feyereislova A, et al.; ToGA Trial Investigators. Trastuzumab in combination with chemotherapy versus chemotherapy alone for treatment of HER2-positive advanced gastric or gastro-oesophageal junction cancer (ToGA): a Phase 3, open-label, randomised controlled trial. Lancet. 2010;376(9742):687-697. doi:10.1016/S0140-6736(10)61121-X.

4. Fuchs CS, Doi T, Jang RW, et al. Safety and efficacy of pembrolizumab monotherapy in patients with previously treated advanced gastric and gastroesophageal junction cancer: phase 2 clinical KEYNOTE-059 trial. JAMA Oncol. 2018;4(5):e180013. doi:10. 1001/jamaoncol.2018.0013

5. Fuchs CS, Tomasek J, Yong CJ, et al.; REGARD Trial Investigators. Ramucirumab monotherapy for previously treated advanced gastric or gastro-oesophageal junction adenocarcinoma (REGARD): an international, randomised, multicentre, placebo-controlled, phase 3 trial. Lancet. 2014;383(9911):31-39. doi:10.1016/S0140-6736(13) 61719-5.

6. Li J, Qin S, Xu J, et al. Randomized, double-blind, placebo-controlled phase III trial of apatinib in patients with chemotherapy-refractory advanced or metastatic adenocarcinoma of the stomach or gastroesophageal junction. J Clin Oncol. 2016;34 (13):1448-1454. doi:10.1200/JCO.2015.63.5995

7. U.S. Food and Drug Administration. FDA appoves trastuzumab (Herceptin); 2010. Available from: http://www.fda.gov/AboutFDA/ CentersOffices/OfficeofMedicalProductsandTobacco/CDER/ ucm230418.htm. Accessed August 3, 2021.

8. U.S. Food and Drug Administration. Ramucirumab in combination with paclitaxel;.2014. Available from: http://www.fda.gov/Drugs/ InformationOnDrugs/ApprovedDrugs/ucm421930.htm. Accessed August 3, 2021.

9. U.S. Food and Drug Administration. Ramucirumab;.2014. Available from: http://www.fda.gov/Drugs/InformationOnDrugs/ ApprovedDrugs/ucm394260.htm. Accessed August 3, 2021.

10. U.S. Food and Drug Administration. FDA grants accelerated approval to pembrolizumab for first tissue/site agnostic indication;.2017. Available from: https://www.fda.gov/drugs/informa tionondrugs/approveddrugs/ucm560040.htm. Accessed August 3, 2021.

11. U.S. Food and Drug Administration. FDA grants accelerated approval to pembrolizumab for advanced gastric cancer;.2017. Available from: https://www.fda.gov/Drugs/InformationOnDrugs/ ApprovedDrugs/ucm577093.htm. Accessed August 3, 2021.

12. Wainberg ZA, Yoon H, Catenacci DVT, et al. Efficacy and safety of pembrolizumab (pembro) alone or in combination with chemotherapy (chemo) in patients (pts) with advanced gastric or gastroesophageal (G/GEJ) cancer: long-term follow up from KEYNOTE-059. J Clin Oncol. 2019;37(15_suppl):4009. doi:10.1200/JCO.2019.37.15_suppl. 4009
13. Shitara K, Özgüroğlu M, Bang YJ, et al.; KEYNOTE-061 investigators. Pembrolizumab versus paclitaxel for previously treated, advanced gastric or gastro-oesophageal junction cancer (KEYNOTE-061): a randomised, open-label, controlled, phase 3 trial. Lancet. 2018;392 (10142):123-133. doi:10.1016/S0140-6736(18)31257-1

14. Ohtsu A, Shah MA, Van Cutsem E, et al. Bevacizumab in combination with chemotherapy as first-line therapy in advanced gastric cancer: a randomized, double-blind, placebo-controlled phase III study. J Clin Oncol. 2011;29(30):3968-3976. doi:10.1200/JCO.2011.36.2236

15. Wilke H, Muro K, Van CE, et al.; RAINBOW Study Group. Ramucirumab plus paclitaxel versus placebo plus paclitaxel in patients with previously treated advanced gastric or gastro-oesophageal junction adenocarcinoma (RAINBOW): a double-blind, randomised phase 3 trial. Lancet Oncol. 2014;15 (11):1224-1235. doi:10.1016/S1470-2045(14)70420-6.

16. Li J, Qin S, Xu J, et al. Apatinib for chemotherapy-refractory advanced metastatic gastric cancer: results from a randomized, placebo-controlled, parallel-arm, phase II trial. J Clin Oncol. 2013;31(26):3219-3225. doi:10.1200/JCO.2013.48.8585

17. Available from: http://www.most.gov.cn/kjbgz/201411/t20141115 116607.htm. Accessed August 3, 2021.

18. Aoyama T, Yoshikawa T. Targeted therapy: apatinib - new third-line option for refractory gastric or GEJ cancer. Nat Rev Clin Oncol. 2016;13(5):268-270. doi:10.1038/nrclinonc.2016.53

19. Chen W, Zheng R, Baade PD, et al. Cancer statistics in China, 2015. CA Cancer J Clin. 2016;66(2):115-132. doi:10.3322/caac.21338

20. Matthaiou C, Papamichael D. Management of gastric cancer in older adults. $J$ Geriatr Oncol. 2017;8(6):403-406. doi:10.1016/j. jgo.2017.07.009

21. Pelucchi $\mathrm{C}$, Lunet N, Boccia $\mathrm{S}$, et al. The stomach cancer pooling (StoP) project: study design and presentation. Eur J Cancer Prev. 2015;24(1):16-23. doi:10.1097/CEJ.0000000000000017

22. Lewis JH, Kilgore ML, Goldman DP, et al. Participation of patients 65 years of age or older in cancer clinical trials. J Clin Oncol. 2003;21(7):1383-1389. doi:10.1200/JCO.2003.08.010

23. Murthy VH, Krumholz HM, Gross CP. Participation in cancer clinical trials: race-, sex-, and age-based disparities. JAMA. 2004;291 (22):2720-2726. doi:10.1001/jama.291.22.2720

24. Kim HS, Kim JH, Kim JW, Kim BC. Chemotherapy in elderly patients with gastric cancer. J Cancer. 2016;7(1):88-94. doi:10.7150/jca.13248

25. Muro K, Cho JY, Bodoky G, et al. Age does not influence efficacy of ramucirumab in advanced gastric cancer: subgroup analyses of REGARD and RAINBOW. J Gastroenterol Hepatol. 2018;33 (4):814-824. doi:10.1111/jgh.14007

26. Kang YK, Kang WK, Di Bartolomeo M, et al. Randomized phase III ANGEL study of rivoceranib (apatinib)+ best supportive care (BSC) vs placebo + BSC in patients with advanced/metastatic gastric cancer who failed $\geq 2$ prior chemotherapy regimens. Ann Oncol. 2019;30: v877-v878. doi:10.1093/annonc/mdz394.034

27. Wang X, Zhang R, Du N, et al. An open label, multicenter, noninterventional study of apatinib in advanced gastric cancer patients (AHEAD-G202). Ther Adv Med Oncol. 2020;12:1758835920905424. doi:10.1177/1758835920905424

28. Wang X, Yu J, Yang M, et al. Safety and effectiveness of apatinib in patients with previously treated metastatic gastric cancer: a sub-analysis from the real-world study of apatinib for gastric cancer treatment (AHEAD-G202). Am J Cancer Res. 2020;10:987-996. 
Video abstract

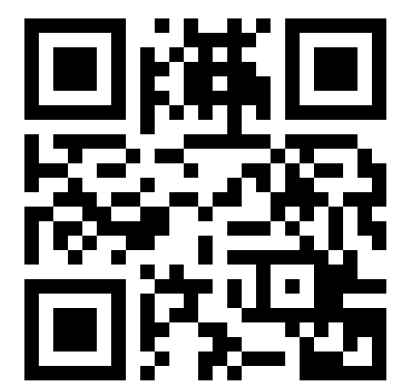

Point your SmartPhone at the code above. If you have a $Q R$ code reader the video abstract will appear. Or use: https://youtu.be/FATzhtdXGn4

\section{Publish your work in this journal}

OncoTargets and Therapy is an international, peer-reviewed, open access journal focusing on the pathological basis of all cancers, potential targets for therapy and treatment protocols employed to improve the management of cancer patients. The journal also focuses on the impact of management programs and new therapeutic agents and protocols on patient perspectives such as quality of life, adherence and satisfaction. The manuscript management system is completely online and includes a very quick and fair peer-review system, which is all easy to use. Visit http://www.dovepress.com/ testimonials.php to read real quotes from published authors. 